\title{
Giant Myofibroblastoma of the Breast: A Case Report and Clinicopathological Analysis
}

\author{
Haizhi Qiao ${ }^{1,2}$, Minghan Yang, ${ }^{1,}$, Jinku Zhang ${ }^{1,2 *}$, Jirui Sun ${ }^{1,2 *}$ \\ ${ }^{1}$ Department of Pathology, Baoding's First Central Hospital, Baoding 071000, China \\ ${ }^{2}$ Hebei Key Laboratory of Molecular Pathology and Early Diagnosis of Cancer, Baoding 071000, China \\ *Jinku Zhang and Jirui Sun are both the corresponding authors and contributed equally to this work.
}

Funding: The Beijing-Tianjin-Hebei Basic Research Cooperation Special Project (2019): "Visualized Stem Cell Targeted Tumor Therapy Techniques for Precise Diagnosis and Treatment of Tumors" Project Number: 19JCZDJC65800(Z).

\begin{abstract}
Myofibroblastoma of the breast (MFB) is a rare benign mesenchymal tumor of the breast. A case of giant breast myofibroblastic tumor, which is rarely reported in literature, was recently diagnosed in our department. We also analyzed the clinicopathological features of MFB to improve the understanding of the tumor and avoid misdiagnosis.
\end{abstract}

Key words: Myofibroblastoma of the breast; Clinicopathological Analysis

Publication date: May, 2021; Publication online: 31 May, 2021

*Corresponding author: Jinku Zhang, 843561234@qq.com; Jirui Sun, 15833867775a@sina.com

\section{Clinical data}

A 34 years old female patient was admitted to hospital on January 12, 2021 because of "right breast tumor was found for more than 6 months, ulceration and pus for more than 1 month". The patient accidentally touched an "egg size" tumor on his right breast more than 6 months ago, and there was no local trauma history. There was no skin redness, swelling, heat and pain on the surface. A small amount of white milk like discharge could be seen after bilateral nipple extrusion. The amount was small and there was no peculiar smell. In December 2020, the tumor increased significantly, and the right breast was broken and purulent in many places, showing yellow white and no abnormal odor. Later, on January 9, 2021, he went to the county hospital for breast ultrasound examination. The results showed that the right breast was enlarged, with large irregular hypoechoic mass in it, which could not be detected. The probe pressure seemed to have peristalsis, with a little punctate blood flow signal in it. The gland was not clearly displayed, and the nipple was damaged Pressure shift. The nature of the huge tumor in the right breast of the patient is yet to be determined. It is suggested that he should be hospitalized. Today, for further diagnosis and treatment, he came to our hospital.

Physical examination was performed after admission. The right breast is large, the right breast is asymmetric, the right breast skin can see multiple ulceration points, has healed, no pus outflow, right nipple deviation, left nipple deviation, double nipple partial invagination, a small amount of white milk like discharge can be seen after squeezing the bilateral nipples, small amount, no peculiar smell, the middle and upper quadrants of the right breast are obviously uplifted, soft, the left breast does not touch the tumor, and the bilateral axillary lymph nodes do not touch. No enlarged lymph nodes were found on the bilateral clavicles.

In order to clarify the nature of the tumor, the patient underwent right breast tumor resection + nipple areola plastic surgery + random flap formation under general anesthesia on January 20, 2021.The size of the tumor was 31 $\mathrm{x} 17.5 \times 11 \mathrm{~cm}$, and the skin area was $15 \times 8 \mathrm{~cm}$. There were 
two ulcers on the surface of the skin. The diameters of the ulcers were $1 \mathrm{~cm}$ and $1.2 \mathrm{~cm}$, respectively. The tumor was complete and the boundary was clear (Figure 1). The solid gray matter on the section was slightly tough. Microscopically, the boundary of the tumor was clear and the edge was pushing (Figure 2). The tumor showed mild spindle cell proliferation, some cells were myofibroblast like, the cytoplasm was eosinophilic, and the nucleus was oval to spindle, which was distributed in the significantly

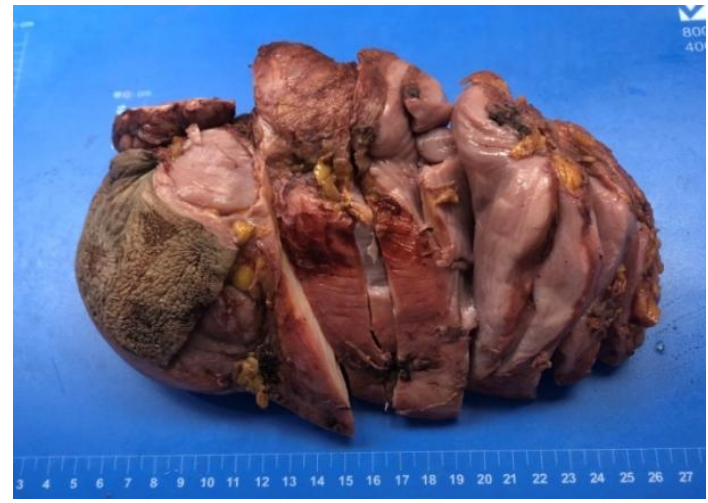

Figure 1. The tumor is well circumscribed

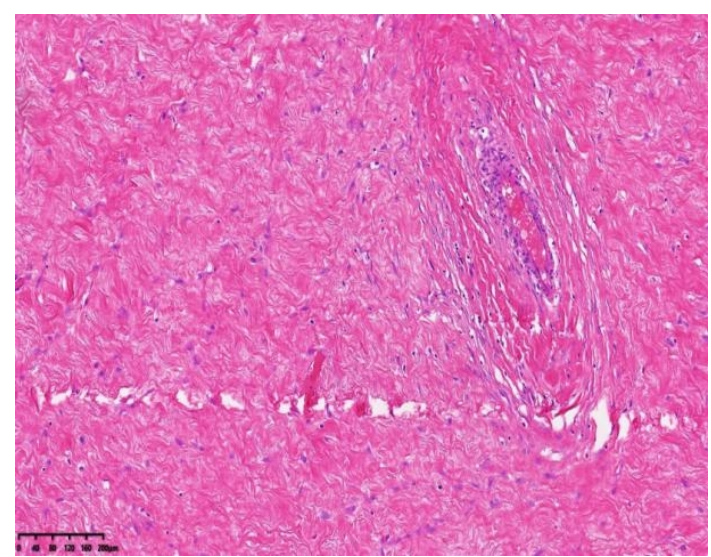

Figure 3. Spindle cell proliferation in collagenated stroma of HE 100x

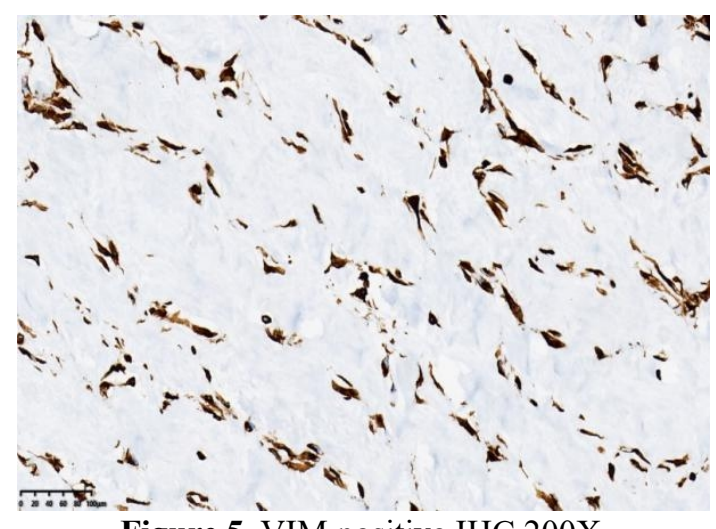

Figure 5. VIM positive IHC 200X collagenous stroma (Figure 3, 4).The routine pathological diagnosis was as follows(right) breast myofibroblastoma, further molecular detection is recommended. Immunohistochemistry was usedCK (-), CK5 / 6 (-), p63 (-), vimentin $(+)$ (Figure 5), SMA (local + ) (Figure 6), ER (-), PR (-), CerbB-2 (-), CD34 (-), Ki67 (1\%), bcl-2 (-), desmin $(-)$, CD31 (-), ERG (vascular endothelium +), EMA (-). The patients were further diagnosed by molecular pathologyRB1 / $13 q 14$ deletion.

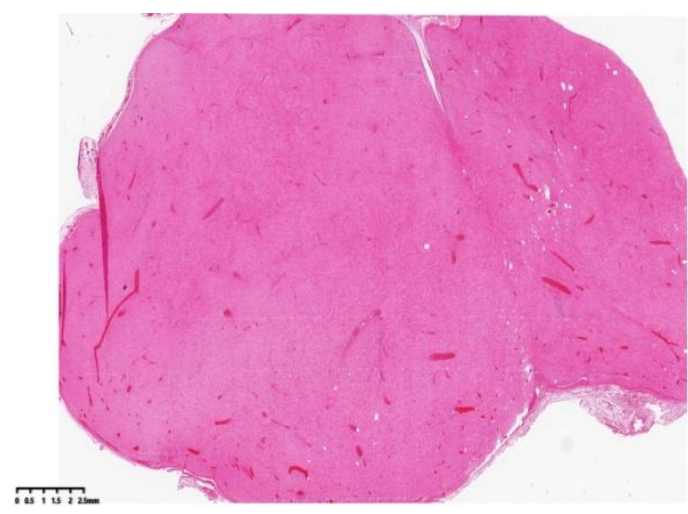

Figure 2. HE 2x tumor is well circumscribed and its margin is pushing

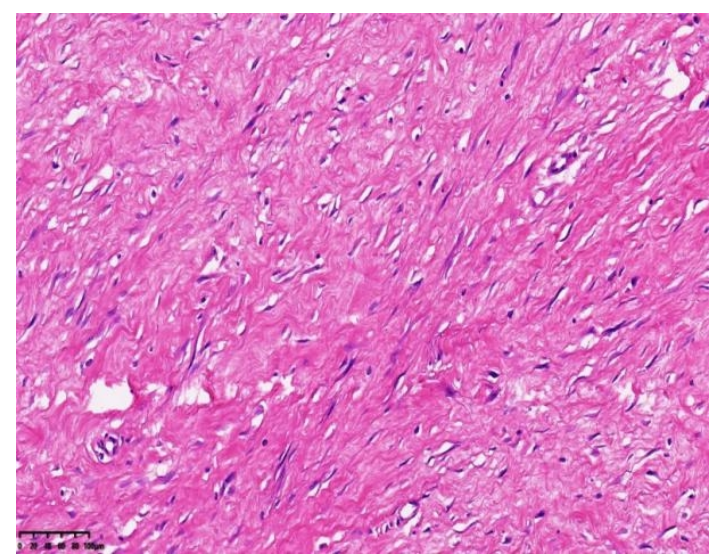

Figure 4. The morphology of HE 200X spindle tumor cells is mild

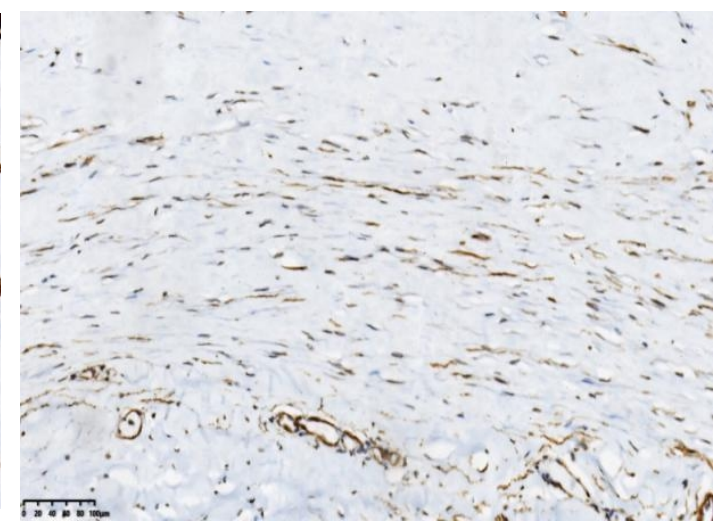

Figure 6. SMA local positive IHC 200X 


\section{Clinicopathological analysis}

Myofibroblastic tumor of the breast (MFB), first named by Wargotz et $a l^{[1]}$ in 1987 , is a benign tumor of mesenchymal origin. It has the characteristics of fibroblasts and smooth muscle cells, which is very rare in clinical diagnosis. The onset age of the disease is wide, which can be seen in adults aged 25-87. There is no gender difference in patients, and it is more common in elderly men or postmenopausal women $^{[2]}$.

Most of the clinical manifestations are solid, painless and movable hard nodules of unilateral breast, which grow slowly. The course of disease varies from several months to several years. It may also grow rapidly in a short period of time. It is rare for bilateral or unilateral multiple lesions. Ultrasound examination often showed irregular hypoechoic nodules, unclear boundary, uneven internal echo, CDFI visible blood flow signal ${ }^{[3]}$.

The tumor size ranged from several millimeters to 11 centimeters (average $2 \mathrm{~cm}$, most $<4 \mathrm{~cm}$ ). Most of the tumors were well circumscribed, non encapsulated masses with smooth edges or lobulated, hard and tough texture ${ }^{[4]}$. Microscopically, the classic type of breast myofibroblastic tumor was characterized by the edge of the tumor was pushed, no true capsule, the tumor was composed of spindle cells, light stained or eosinophilic cytoplasm, mild nucleus, oval to spindle shape, arranged in short bundles, mixed with characteristic scar like collagen fiber bundles, fat, smooth muscle, cartilage or bone tissue components can be seen in the tumor, and mucoid degeneration can be seen in the stroma Mitotic figures were very rare ${ }^{[5]}$. There were six variants of myofibroblastoma. (1) Lipomatous typeIt was composed of mature fat components (accounting for more than $50 \%$ of the whole tumor), in which nodular or irregular fibrous areas were scattered, and the typical features of myofibroblastic tumor can be recognized locally in the fibrous area ${ }^{[6]}$. (2) Mucoid type. The tumor was completely or mainly composed of myxoid stroma, with spindle and stellate cells distributed in myxoid stroma $^{[7]}$. Fibrosis/collagen type. Spindle cells were few and distributed in the diffuse fibrosclerotic stroma, with irregular fissure like spaces of pseudoangiomatous stroma hyperplasia ${ }^{[4]}$. (4) Epithelioid type. Tumors mainly composed of medium-sized cells with epithelioid morphology ( $>50 \%$ ), The nuclei of the tumor were round, oval, mononuclear or binuclear, and the cytoplasm was deeply eosinophilic. The tumor cells were arranged in various growth patterns, including nest, solid sheet, micronodule, acinar or trabecular. The tumor cells can be arranged in a single cell or a single row of cord structure, which was very similar to invasive lobular carcinoma, and the stroma was fibrotic or mucoid ${ }^{[8]}$. (5) Decidual type. Tumor cells were similar to decidua like morphology, with abundant cytoplasm, eosinophilic, large nucleus, vacuole like and obvious nucleolus ${ }^{[9]}$. (6) Palisade / neurilemmoma like. It was composed of single spindle cells with palisade like nuclei, forming many Verocay like bodies ${ }^{[10]}$.

Immunohistochemical staining showed that tumor cells expressed vimentin, desmin (positive rate 89\%) and CD34 (positive rate $91 \%)^{[11]}$. Some cases expressed Er, PR and AR, and myogenic markers such as SMA and h-caldesmon were expressed in varying degrees. There was no expression of CK, EMA, HMB-45, CD117 and S-100 ${ }^{[12]}$. Cytogenetic studies have shown that breast myofibroblastic tumor is a tumor with deletion of chromosome 13. Recent FISH studies have shown that it is characterized by single or double allele deletion of RB1 / 13q14 and / or single allele deletion of fox1 / 13q14 ${ }^{[4]}$. A few cases showed chromosome 16 deletion.

In this case, the boundary of the tumor was clear. The fibroid spindle cell proliferation, eosinophilic cytoplasm and oval to spindle nucleus were seen in the stroma with significant collagenation, which was consistent with the microscopic pathological features of myofibroblastic tumor (fibrotic / collagenated type). Immunohistochemical results showed vimentin positive, SMA local positive, consistent with the immunohistochemical expression of myofibroblastoma, desmin and CD34 negative, consistent with a few negative cases of breast myofibroblastoma in the literature, Ki67 proliferation index was $1 \%$, tumor cells $\mathrm{CK}$, CK5 / 6, S-100, er, PR were negative, spindle cell metaplasia carcinoma and neuro adipose derived tumors can be excluded. The results of tumor molecular detection showed that: 1RB1 / 13q14 deletion supports the cytogenetic characteristics of breast myofibroblastoma. This case was diagnosed as myofibroblastic tumor (fibrotic / collagenous type) according to the history, histological morphology, immunohistochemical results and molecular detection results. The largest diameter of the tumor is $31 \mathrm{~cm}$. It is a giant myofibroblastic tumor of the breast, which is rarely reported in the literature at home and abroad.

Local complete resection of breast myofibroblasts can 
be cured, and the recurrence and metastasis rates are low ${ }^{[13]}$. It is easy to be misdiagnosed in the intraoperative pathological diagnosis, so it needs to be differentiated from spindle cell proliferative lesions. (1) Pseudoangiomatous stromal hyperplasia (PASH): Under the bundle type microscope, myofibroblasts gathered in bundles and pseudovascular spaces disappeared, similar to myofibroblastoma, but different from breast myofibroblastoma, the proliferation of PASH included perilobular and intralobular stroma, including mammary duct / lobule ${ }^{[14]}$. (2) Reactive spindle cell nodules. It is the proliferation of myofibroblasts produced by tissue injury, previous history of biopsy or fine needle aspiration, phagocytosis of hemosiderin cells, lymphocytes, plasma cells, fat necrosis and other reactive changes, which support the morphological characteristics of the lesion. (3) Solitary fibrous tumor (SFT) overlaps with breast myofibroblastic tumor in morphology and immunohistochemistry, and can express CD34. However, the characteristic nuclear expression of SFT is STAT6, which is usually desmin negative, and $\mathrm{Rb}$ is absent ${ }^{[11]}$. (4) Others: Desmoid fibromatosis, lobular tumor, invasive lobular carcinoma, spindle cell metaplasia carcinoma, etc. Therefore, it is necessary for pathologists to improve the accuracy of pathological diagnosis on the basis of accumulated diagnosis experience, combined with immunohistochemistry and molecular pathology, so as to avoid misdiagnosis.

\section{References}

[1] Wargotz ES, Weiss SW, Norris HJ. Myofibroblastoma of the breast.Sixteen cases of a distinctive benign mesenchymal tumor [J]. Am J Surg Pathol, 1987, 11(7): 493-502.

[2] Qin R, Zhang RY. Benign breast lesions easily misdiagnosed as malignant tumors [J]. Journal of Jining Medical College, 2019, 42 (5): 321-326 + 331.

[3] $\mathrm{Xu} \mathrm{ML}, \mathrm{Li} F$, Zeng S. Ultrasound findings of breast myofibroblastic tumor: one case [J]. Chinese Journal of Ultrasound Medicine, 2020, 36 (5): 479.
[4] Yu BH, Bai QM, Xu XL, et al. Clinicopathological analysis of nine cases of breast myofibroblastic tumor [J]. Chinese Journal of Pathology, 2018, 47(10): 747-752.

[5] Krings G, McIntire P, Shin SJ. Myofibroblastic, fibroblas-tic and myoid lesions of the breast [J]. Semin Diagn Pathol, 2017, 34(5): 427-437.

[6] Magro G, Michal M, Vasquez E, \& Bisceglia M (2000). Lipomatous myofibroblastoma: A potential diagnostic pitfall in the spectrum of the spindle cell lesions of the breast. Virchows Archiv, 437, 540-544.

[7] Magro G, Amico P, \& Gurrera A (2007). Myxoid myofibroblastoma of the breast with atypical cells: A potential diagnostic pitfall. Virchows Archiv, 450, 483-485.

[8] Magro G, Vechio GM, Michal M, et al. Atypical epithelioid cell myofibroblastoma of the breast with multinodular growth patten;a potential pitfall of malignancy $[\mathrm{J}]$. Pathol Res Pract, 2013, 209(7): 463-466. DOI: 10.1016.

[9] Magro G (2008). Mammary myofibroblastoma: A tumor with a wide morphologic spectrum.

[10] Magro G, Foschini M, M.P, \& Euscbi V (2013). Palisaded myofibroblastoma of the breast:a tumer clsely mimicking schwannoma. Report of two cases.Human Pathology,44,1941-1946.

[11] Wang J, Zhu XZ. Pathology of Soft Tissue Tumor [M]. Beijing: People's Health Publishing House, 2017:271-274.

[12] Zhu HM, Liu JP, Guo ZY. Breast myofibroblastic tumor: a case report and literature review $[\mathrm{J}]$. Chinese Journal of Cancer Prevention and Treatment, 2017,24 (24): 1747-1750.

[13] Howitt BE, Fletcher CD. Mammary-type myofibroblastoma:clinicopathologic characterization in a series of 143 cases [J]. Am J Surg Pathol, 2016, 40(3): 361-367.

[14] Virk R K,\& Khan A (2010). Pseudoangiomatous stromal hyperplasia an overview [J]. Archives of Pathology \& Laboratory Medicine, 134: 1070-1074. 